\title{
Research on the Fusion of Art Design and Screen Printing Process
}

\author{
Jun $\mathrm{CaO}^{1, *}$ \\ ${ }^{1}$ Nanjing City Vocational College, Nanjing, Jiangsu 210000, China \\ *Corresponding author.Email:2021006@ncc.edu.cn
}

\begin{abstract}
Starting from the classification, concepts and creative techniques of art design form language, this author of this paper analyzes the main characteristics and aesthetic value of different art design forms, and through practice, closely integrates art design and screen printing process, so that art design works presented a more novel and unique style under the process of screen printing, and also confirms the important role and far-reaching influence of screen printing on art design. The integration of the two has important theoretical guiding significance and practical value for studying the creative methods of different art design form languages, insighting into the restrictive relationship between art design performance and screen printing, and mastering the originality and technology of art design.
\end{abstract}

Keywords: Art design, Screen printing process, Morphological language, Fusion.

\section{INTRODUCTION}

With the development of economy and society, people are increasingly pursuing a better life. Not only have they raised their requirements for material civilization, they have also pursued cultural and artistic aesthetics, especially the daily necessities and craft supplies that are closely related to life. With the rapid progress of science and technology in China, the quality of various products is not only getting better and better, but the prices are getting cheaper. However, the development of culture and art in China is still lagging behind the development of natural science. The main reason is that the development of science and technology is manifested in the innovation of technology and craftsmanship, independent of social science, and lack of integration of art and design. Therefore, how to better integrate art and craftsmanship, enhance the cultural flavor and artistic beauty of industrial products, and meet the growing cultural and artistic needs are still urgent problems to be solved. This article starts from the research on the relationship between the screen printing process

*Project: This article is one of the research results of the teaching innovation team of the product art design professional group, a key construction project in Nanjing during the "14th Five-Year Plan". and the artistic design, and discusses the integration and development of process and design.

Screen printing originated from leak printing in the Qin and Han dynasties, and developed from clip printing in the Eastern Han Dynasty to screen printing in the Sui Dynasty. In the Song Dynasty, people greatly improved dyes and increased the vividness of printed colors. After the Qing dynasty, screen printing technology has made great progress in the West [1]. In the 1980s, the wave of reform and opening up stimulated the influx of large quantities of foreign textile processing orders and material technology, and also spurred the rise of the screen printing industry and the growth of the team of industrial technical workers. At the end of the 20th century and the 21 st century, the development of science and technology has provided excellent equipment and materials for screen printing, promoted the progress of screen printing technology and the perfection of craftsmanship, and made it show a strong "vitality". Screen printing has the advantages of cost economy, easy operation, freedom of expression, and diverse media. Screen printing has provided a medium for the creation of artistic design since its birth. From the simple patterns at the beginning to the complex patterns with various, levels and colors, especially the emergence of various new materials, new 
technical methods have been provided for the screen printing process. The development of new structures of various ink materials provides rich visual art aesthetics for screen printing products. Its combination with art design highlights its strong practical application value and artistic appreciation value, which greatly caters to the individual needs of modern people for design aesthetics. The development of the two complements each other, so that it occupies an important place in the field of art design.

\section{THE EXPRESSION AND APPLICATION OF ARTISTIC DESIGN FORM LANGUAGE IN SCREEN PRINTING PROCESS}

Starting from the noumenon of artistic design form language, cognizing their performance techniques in screen printing can provide insight into the interrelationship between design concepts, design forms and craft materials, so as to better enhance the functionality, decoration and innovation of artistic design works. Based on image, the language of artistic design form can be summarized into three categories: concrete form language, image form language and abstract form language.

\subsection{Concrete Form Language and Its Expression in the Screen Printing Process}

Concrete form language is an imitation of natural objects (plants and animals, people, landscapes) and man-made objects (buildings, tools, utensils). It reflects the specific appearance and posture characteristics of objects, and is realistic and intuitive. [2] In the creation of concrete morphological language, realistic super-realistic expressions can be used to shape objects. Through multi-level and multi-angle observation and depiction, the structure and details of the object can be expressed to the fullest, so as to achieve an incredible simulation effect; the comic cartoons full of fun can also be used to express objects, which can abandon the simple imitation of object modeling, and favor the exaggeration and emphasis on object features and expressions, creating a creative visual image "originating from nature and higher than nature".

In recent years, screen printing has taken great interest in the reproduction of concrete forms on substrates. The development and popularization of computer digital technology has also enabled screen printing to have this ability. "The application of computer digital graphics processing technology has played a huge role in enriching the expression space of printing plates, making it possible to truly reproduce computer-generated works on actual products". [3] To realize a concrete image with rich colors and complex content through the screen printing process, the most commonly used method is to first use computer-aided software to separate the four basic tones of cyan (C), magenta (M), yellow (Y), and black $(\mathrm{K})$ in the image. The computer-aided software separates the image into four CMYK colors, and converts them into dots of different sizes for storage, and then prints the four files with different dots on the film to obtain a higher-quality printing film base. Then people need to follow the conventional process of screen printing, that is, stretching screen - screen pretreatment - coating photosensitive glue, drying - exposure - development, drying [4], and finally use the method of lamination and reorganization to print.

The concrete form can also be realized by hand drawing. People usually use sulphuric acid paper, colored chalk and charcoal strips to draw editions, which can not only improve the creator's cognitive ability, expressive ability and creative ability of artistic design form language, but also add a more natural, soft and varied effect to the work.
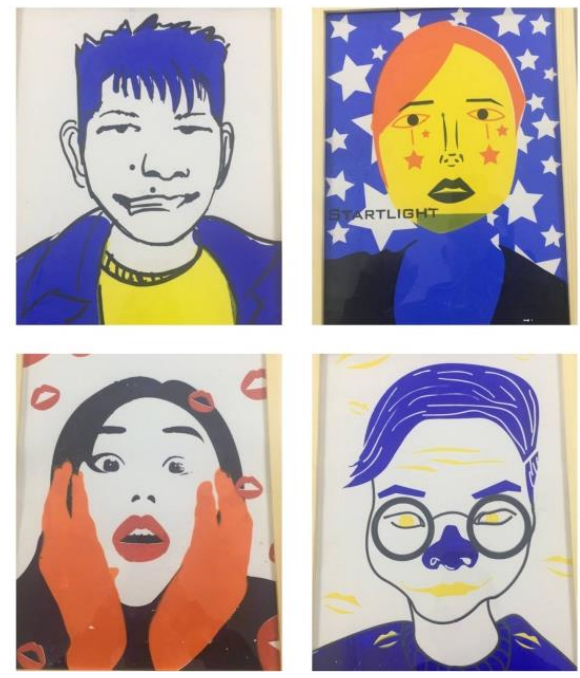

Figure 1 Screen printing works of "Portrait of People".

"Figure 1" is the screen printing works of "Portrait of People". Through careful observation and exaggerated depiction of the characteristics of each character, combined with the unique 
craftsmanship of screen printing, it can present a variety of visual and tactile possibilities, extending the expression space of art design works in screen printing, which also realizes the unity and fun of design and technology.

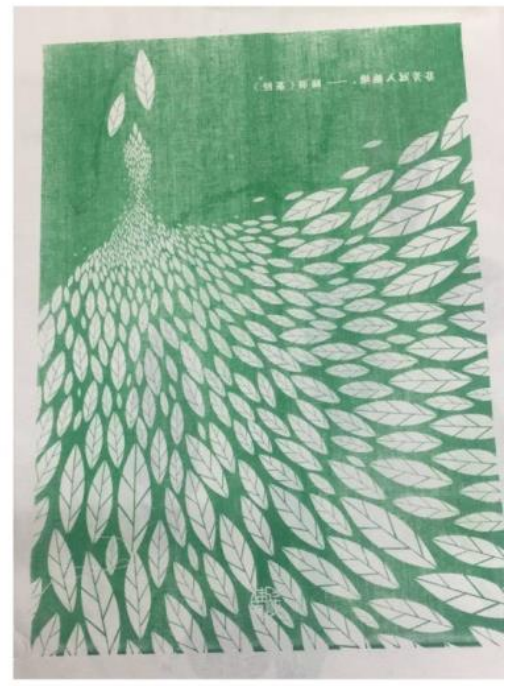

Figure 2 Screen printing poster of "Yixing Tea".

In addition, people can also use decorative forms to create the concrete form of the object, so that the object presents an ideal visual effect. This form of expression often needs to be based on realism and use the law of formal beauty to change the object to make it have the characteristics of the formal beauty on the plane. "Figure 2" shows the screen-printed poster of "Yixing Tea". The design is inspired by the scene of frying tea. The author uses modern modeling concepts and composition methods to extract the concrete form of tea into a cognitive design symbol, and then recombines this symbol to construct a spatial structure rich in threedimensionality and formal beauty. The graphics, logos and explanatory texts in the work are all white, and they are printed in a single color on the textile linen according to the conventional process when printing.

\subsection{Abstract Form Language and Its Expression in the Screen Printing Process}

"The abstract forms in the art design category mainly refer to pure geometric forms, that is, various forms in geometry such as squares, rectangles, triangles, circles and ellipses, irregular geometric shapes and geometric bodies, etc., they can please people with pure forms, and they have to be pictographic at all" [2]. That is to say, the abstract forms do not reflect any concrete objects, nor do they have any concrete content at all. They are purely the combinations of lines, faces and color blocks to obtain the formal beauty of the work. Therefore, when creating abstract works, creators can use their own language to directly express their true inner feelings, and don't have to care whether the "language" they use is like or not like a specific concrete object. The work is a medium for expressing the thought and emotion of the creator. When the audiences interpret abstract works, they can also give full play to their personal imagination without being stuck on a specific theme. The works contain unlimited exploration space and artistic beauty. Abstract form is one of the forms that the screen printing process is good at. In "Figure 3", the color is subdivided into yellow, blue, and black during the plate making process. During printing, the color separation plates are sequentially overprinted on the substrate according to the order of color design, thereby achieving the ideal effect of multi-level and multispace screen printing products.

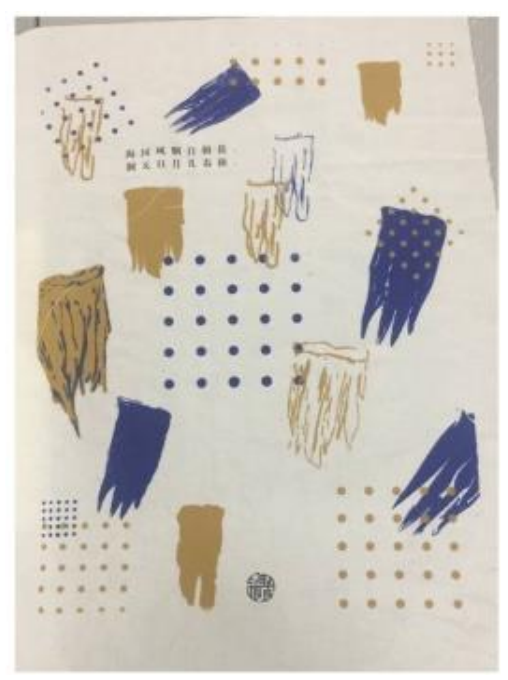

Figure 3 Screen printing works in abstract form.

\subsection{Image Form Language and Its Expression in the Screen Printing Process}

"Image form is a new image produced by objective things reflected in the creator's subjective consciousness and recombined by the creator's brain. It is a perceptual object or form existing in the human mind or thought" [5]. Different from the concrete form, which "always does not deviate from the essence of objective things", the image form is the product of the combination of objective and subjective, reality and fantasy. The creation of 
intentional form can be realized by associating and imagining the shape of objective things, using methods such as isomorphism, substitution, and superposition, to make it a new form that is not the same as objective things, but can be recognized and understood. In addition to changing the external shape of an objective thing, it can also change its internal structure to create a new form. "Figure 4" shows the "Bamboo" screen-printed poster. Through careful observation and in-depth research on bamboo forests, the designer refined bamboo forest, bamboo, bamboo shoots and other elements, and used modern methods of composition, which accurately expresses the lushness and prosperity of bamboo forests, the uprightness and height of bamboo, and the appearance and growth of bamboo shoots. In the screen-making process, the variability and unity of the screen-printed products can be realized through the five-color printing method, and the artistic effect can be enriched.

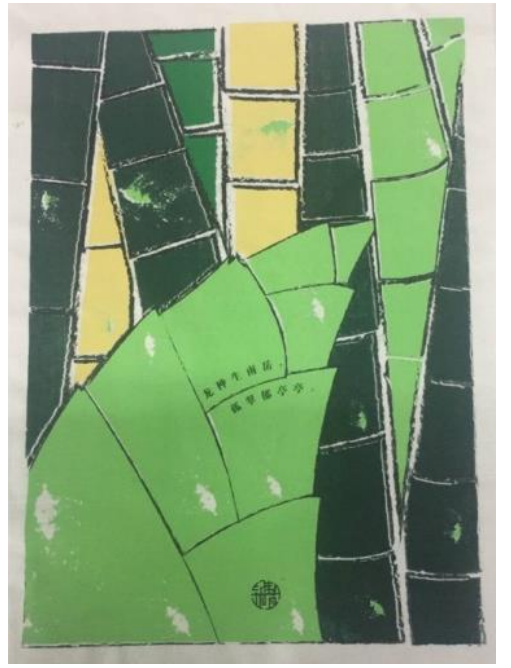

Figure 4 "Bamboo" screen printing poster work.

The creators can use the screen printing process to print monochromatic works to make the picture more concise and strong visual impact; they can make the color of the picture more vivid through the repeated overlay printing method to achieve the desired effect; they can increase the number of chromatographic printing methods to make the picture effect richer; they can reconcile the transparent and semi-transparent printing methods to make the color of the picture lighter, translucent, and more layered; they can add fluorescent pigments to the printing ink to make the picture have fluorescent properties and achieve special effects that other printing processes cannot achieve. In addition, the creators can also try to print on different substrate materials to make the art design work diversified; they can purposefully select the substrate materials according to the purpose of the work to meet the functional requirements of the design; and they can also better convey the form and connotation of the work according to the content of the work, with the help of the characteristics and emotions of the material language.

\section{THE ROLE OF SCREEN PRINTING PROCESS IN PROMOTING ARTISTIC DESIGN}

The screen printing process has a high degree of integration of different artistic design forms and languages. It can not only be used as an important means of artistic design expression, but also endows the vivid charm of artistic design in visual and tactile senses.

First of all, the operating flow of the screen printing process has played a huge role in the personality of the art design. The screen printing process is mainly "manual operation", with screen, ink and substrate as the main materials, and screen frame, squeegee and printing table as the main tools. "During printing, the ink is transferred to the substrate through the mesh of the image and text part through the squeeze of the squeegee, forming the same image and text as the original."[6] This manual printing process has great simplicity, uniqueness and flexibility. On the one hand, it gives artistic design works a rich and diverse visual form. If the angle and speed of the squeegee are different during printing, the color effect of the printed work will also be different; If the ink is printed on different substrates, due to the different materials of the substrates, the ink absorption is also different, making the same image present a rich effect; Another example is the printing of multiple singlepage gradation works. Because the inks of multiple colors are arranged horizontally and mixed on a screen printing plate, and then scratched vertically, the "flexibility" of manual operation gives the work the possibility of various colors. On the other hand, the production process of the screen printing process relies on the operation of the producer's hands to achieve the birth of art and design works, making the works more unique and precious.

Secondly, the screen printing process has a wide range of "printability". It can not only be printed on substrates of different materials and shapes to meet their needs, but it also enhances the artistic charm of these substrates and turns them into art and design products with added value. On the one hand, 
screen printing can not only print on flat objects such as paper, textiles and other conventional materials, but also on curved surfaces, spherical surfaces, or uneven shapes, such as woodware, lacquerware, leather, etc. It is more able to print on the surface of special materials with higher hardness and fragility, such as glass, metal, ceramics and other materials. On the other hand, some substrates have simple shapes and beautiful forms, such as smooth-line ceramics, hard and bright metals, etc.; some substrates have a natural texture on the surface, such as simple and elegant wood, natural mottled leather, etc. These substrates are already independent works of art. Applying the traditional process of screen printing to them not only enriches their functions, but also strengthens their artistic expression, making them extremely have the use value and aesthetic value In addition, the screen printing process has a wide range of substrate sizes. It can not only print on super-largeformat substrates, such as large outdoor advertisements, curtains, etc., so that they have received widespread attention for their wide area and brilliant colors. It can also print on ultra-small and ultra-high-precision items, such as penholders, keyboards, etc., to bring different visual surprises to the audience.

Finally, the screen printing process makes the printed art design works show a unique sense of texture, which enhances the artistic effect and ornamental value of the works. In the design, texture refers to the surface texture of the work, which can sometimes be touched and sometimes seen [2]. The texture effect in screen printing is mainly realized by ink, and it is displayed through the carrier of the substrate. There are many types of screen printing inks, and different types of inks have different properties. They will also produce different texture effects on the substrate, thereby expressing different emotions. People can try to use different types of ink materials to explore different visual and tactile perceptions. For example, after the foaming ink is printed and cooled, when the color base is basically unchanged, it can not only increase the color saturation and brightness of the printed product, but also obtain embossed threedimensional ink clusters and high saturation color effects, highlighting its uneven texture [7]; It is also possible to repeatedly or superimpose the same type of ink on the substrate to make the work have distinctive, three-dimensional and touch characteristics, thereby bringing a pleasant sensory experience to the audience. It is worth mentioning that the texture effect in the screen printing process is accidental and subjective. The unique manual craftsmanship of screen printing gives the producer a larger creative space. The differences in the expression materials, printing media and expression techniques used by the producers will cause the works to show different texture effects. It is this contingency and subjectivity that gives the screenprinted works the vigor and artistic quality, and achieves special effects that other printing processes cannot match.

\section{CONCLUSION}

The screen printing process has a long history and deep accumulation, and it "stands up" in the ups and downs of the years. Integrating screen printing technology with art design can make people recognize the characteristics of different art design forms, master their expression techniques in screen printing, and innovate art design thinking. At the same time, the screen printing process makes the art design work present a unique effect, and also enhances the artistic charm of different substrates, opening up a broad sky for the expression of art design. Research has proved that screen printing, a traditional process that has more than 2,000 years of history, has advantages that other printing processes cannot surpass. It is believed that it can accelerate the individualization, trend and creativity of the art design industry, and win a larger market for the art design industry.

\section{AUTHORS' CONTRIBUTIONS} Cao.

This paper is independently completed by Jun

\section{REFERENCES}

[1] Wei Ping, Analysis on the Development and Production Technology of Screen Print [J]. China Forest Products Industry, 2019, 4. (in Chinese)

[2] Zhuge Kai, Art Design [M]. Shandong Fine Arts Publishing House, 2009, p173-178. (in Chinese)

[3] Qin Ya'na, Digital Technology Brings New Developments in Screen Plate [J]. Art Education Research, 2013, 2. (in Chinese)

[4] Li Yalin, Zhang Jian, Qu Liu, etc. Effect of Screen Printing Technology on the Printing Results of Diatomite Wallpaper [J]. Packaging Engineering, 2014, 23. (in Chinese) 
[5] Li Chengrang, Concrete, Abstract and Image Forms — Three Formal Languages for Interpreting Modeling [J]. Literary Contention, 2010, 4. (in Chinese)

[6] Wang Yuting, Application of Special Printing in Textile Dyeing and Printing Products [J]. Textile Dyeing and Finishing Journal, 2016, 10. (in Chinese)

[7] Chen Shuying, Sun Zhicheng, Li Luhai, etc. Screen Printing Effects and Characteristics of Microsphere Physical Foaming Ink [J]. Packaging Engineering, 2017, 1. (in Chinese) 